\title{
External Magnetic Field Effects on the Rayleigh-Taylor Instability in an Inhomogeneous Rotating Quantum Plasma
}

\author{
G. A. Hoshoudy ${ }^{1,2}$ \\ ${ }^{1}$ Department of Applied Mathematics, Faculty of Science, South Valley University, Qena, Egypt \\ ${ }^{2}$ Faculty of Girls at Muhayel, King Khalid University, Muhayel, Kingdom of Saudi Arabia \\ Email: g_hoshoudy@yahoo.com
}

Received August 29, 2012; revised September 29, 2012; accepted October 8, 2012

\begin{abstract}
The effects of external magnetic field effects on the Rayleigh-Taylor instability in an inhomogeneous stratified quantum plasma rotating uniformly are investigated. The external magnetic field is considered in both horizontal and vertical direction. The linear growth rate is derived for the case where a plasma with exponential density distribution is confined between two rigid planes at $z=0$ and $z=h$, by solving the linear QMHD equations into normal mode. Some special cases are particularized to explain the roles that play the variables of the problem. The results show that, the presence of both external horizontal and vertical magnetic field beside the quantum effect will bring about more stability on the growth rate of unstable configuration. The maximum stability will happen in the case of wave number parallels to or in the same direction of external horizontal magnetic field.
\end{abstract}

Keywords: Rayleigh-Taylor Instability; Quantum Plasmas; Magnetic Field

\section{Introduction}

when you hear the word "plasma", what do you think of? Do you think of the sun, lightning bolts or fluorescent lamps? Or do you think of nuclear fusion, micro processsor manufacture or lasers? while naturally occurring plasma is relatively unusual on earth it is playing a larger and increasingly important role in how we use and develop modern technology. For instance, producing compact computer chips on an industrial scale is only made possible by the application of plasma. Plasma is also a key technology in the development of alternative energy sources. Nuclear fusion, which is plasma based, is one of the most promising candidates for the energy needs of the future when fossil fuels finally run out. Plasma is increasingly becoming part of the industrial arena and its range of application is vast. The different variables of plasma play important role in the general behavior of the considerable model. The pressure one of this variables, that is divided to two term $P=P^{C}+P^{Q}$ (Classical $\left(P^{C}\right)$ and quantum $\left(P^{Q}\right)$ pressure) $[1,2]$. In the momentum equation the classical pressure rises in the form $(-\nabla P)$, while the quantum pressure rises in the form $\boldsymbol{Q}=\frac{\hbar^{2}}{2 m_{e} m_{i}} \rho \nabla\left(\frac{\nabla^{2} \sqrt{\rho}}{\sqrt{\rho}}\right)$, where $\hbar$ is the Plank constant, $m_{e}$ is the electron mass and $m_{i}$ the ion mass. One of the important model that rises in hydrodynamic plasma that is called the Rayleigh-Taylor instability problem $[3,4]$. Where the Rayleigh-Taylor instability, in magnetized plasma, can occur because the magnetic field acts as a light fluid supporting a heavy fluid (plasma). In curved magnetic fields, the centrifugal force on the plasma due to particle motion along the curved lines acts as an equivalent gravitational force. The hydro-magnetic stability of magnetized plasma of variable density is of considerable importance in several astrophysical situations, e.g. in theories of sunspot magnetic fields, heating of solar corona and the stability of stellar atom-spheres in magnetic fields. The appearance of such instabilities in previous topics has inspired us to study it and this is the main motivation of this work. The instability of stratified plasmas in the classical case, in the presence of vertical and horizontal magnetic field of compressible plasmas, respectively, is studied by Bhimsen [5] and Liberatore et al. [6]. In the presence of magnitude of the gravitational acceleration $g$, the instability of stratified incompressible plasmas was studied by Goldston and Rutherford [7] and in the presence of horizontal magnetic field of plasmas it was studied by Wu et al. [8].

Currently, there is a rapidly growing interest in the field of quantum plasmas, where quantum plasmas have 
a wide range of application [9-15]. Quantum plasmas plays an important role in ultra small electronic devices [9], dense astrophysical plasmas system [10,11], intense laser-matter experiments [12], and nonlinear quantum optics $[13,14]$.The quantum effects become important in the behavior of charged plasma particles when the de Broglie wavelength of charge carriers become equal to or greater than the dimension of the quantum plasma system [15]. Quantum plasmas can be composed of electrons, ions, positrons, holes and/or grains. Two models are used to study quantum plasmas systems. The first one is the Wigner-Poisson and the other is the SchrödingerPoisson approaches $[15,16]$ they have been widely used to describe the statistical and hydrodynamic behavior of the plasma particles at quantum scales in quantum plasmas. The quantum hydrodynamic model was introduced in semiconductor physics to describe the transport of charge, momentum and energy in plasma [17]. Several studies were analyzed both analytically and numerically in plasmas with quantum corrections. For example, Haas et al. [18] studied a quantum multi-stream model for one and two stream plasmas instabilities. Bengt Eliasson et al. [19] and by employing the Wigner-Poisson model they studied the dispersion properties of electrostatic oscillations in quantum plasmas for different parameters ranging from semiconductor plasmas to typical metallic electron densities and densities corresponding to compressed matter and dense astrophysical objects. Haas [2] extended the QHD equations for quantum magneto-plasmas and presented a magneto-hydrodynamic (QMHD) model by using the Wigner-Maxwell equations. A range of classical subject such as waves and stream instability (as Jeans instability [20] and Rayleigh-Taylor instability [3,4]) were studied again in quantum plasmas system. Currently, there are many studies on hydrodynamic instabilities in quantum plasmas, in particular RTI [21-30]. For example, the quantum effects on the internal waves and the RTI in plasma is studied by Bychkov et al. [21]. The effect of quantum mechanism on RTI in the presence of horizontal magnetic field was studied by Jintao et al. [22], while Hoshoudy studied the same model in the presence of the vertical magnetic field $[23,24]$. The effects of quantum term on RTI in the presence of vertical or horizontal magnetic field of rotating plasma are studied by Hoshoudy $[25,26]$. RTI in quantum plasmas with para-and ferromagnetic properties is studied by Modestov et al. [27]. The effect of quantum term on RTI of stratified plasma layer through porous medium is studied by Hoshoudy $[28,29]$.The RTI is studied in a non-uniform dense quantum magneto-plasma by Ali et al. [30].

In this paper the effect of quantum term on RTI of stratified plasmas layer in the presence of external magnetic field (horizontal and vertical) is considered. The normalized growth rate as a function of the physical pa- rameters of the problem is obtained and analyzed.

\section{Fundamental Equations}

Our starting point is the system of equations describing the hydrodynamic motion of quantum rotating plasma as a fluid of electrons and immobile ions, where the plasma action by a constant magnetic field along the $y$ and z-axis (i.e. $\quad \boldsymbol{B}=B_{0 y} \mathbf{e}_{y}+B_{0 z} \mathbf{e}_{z}$ ) (see Refs. [22-30])

$$
\begin{aligned}
& \left(\frac{\partial}{\partial t}+\boldsymbol{U} \cdot \nabla\right) \rho \boldsymbol{U}=-\nabla P+\rho \boldsymbol{g}-(\boldsymbol{U} x \boldsymbol{\omega})+\boldsymbol{Q}, \\
& \frac{\partial \rho}{\partial t}+\nabla \cdot(\rho \boldsymbol{U})=0 .
\end{aligned}
$$

Here $\boldsymbol{U}$ is the velocity of the fluid, $\rho$ is the density, $p$ thermal pressure, $\boldsymbol{g}$ is the gravitational acceleration and $\boldsymbol{\omega}=\boldsymbol{\omega}_{y}+\omega_{z}=\left(\omega_{y} \mathbf{e}_{y}+\omega_{z} \mathbf{e}_{z}\right)=\frac{e \boldsymbol{B}}{m_{e}}$ is the plasma angular velocity and $e$ is magnitude of the electrons charge.

To investigate the stability of hydrodynamic motion, we ask how the motion responds to a small fluctuation in the value of any of the flow variables appearing in the Euler equations. If the fluctuation grows in amplitude so that the flow never returns to its initial state, we say that the flow is unstable with respect to fluctuations of that type. Accordingly, we replace the variables in Equations (1) and (2) as follows:

$$
\boldsymbol{U}=\boldsymbol{U}_{0}+\boldsymbol{U}_{1}, \rho=\rho_{0}+\rho_{1}, p=p_{0}+p_{1}, \boldsymbol{Q}=\boldsymbol{Q}_{0}+\boldsymbol{Q}_{1} .
$$

The quantities with subscripts " 0 " represent the unperturbed, or "zeroth-order" motion of the fluid, while the quantities with subscripts "1" represent a small perturbation about the zeroth-order quantities (first-order or linearized quantities); that is, $\left|U_{1}\right| \ll\left|U_{0}\right|,\left|\rho_{1}\right| \ll\left|\rho_{0}\right|$, $\left|p_{1}\right| \ll\left|p_{0}\right|$ and $\left|Q_{1}\right| \ll\left|Q_{0}\right|$. Substituting these expressions into Equations (1) and (2). In particular example of RTI we consider the fluid is initially at rest. This means that $\boldsymbol{U}_{0}=0$. So, the hydrodynamic equilibrium (equilibrium or equation state) is determined by the balance of pressure, gravity and influence of the Bohm potential $\left(\frac{\mathrm{d} p_{0}}{\mathrm{~d} z}=-\rho_{0} g+\frac{\hbar^{2}}{2 m_{e} m_{i}} \rho_{0} \frac{\mathrm{d}}{\mathrm{d} z}\left\{\frac{1}{\sqrt{\rho_{0}}} \frac{\mathrm{d}^{2} \sqrt{\rho_{0}}}{\mathrm{dz} z^{2}}\right\}\right)$ and it does not necessarily imply thermodynamic equilibrium, while the relevant linearization perturbation equations may be written as (see Refs. [22-28]) from Equations (1) and (2) as

$$
\begin{aligned}
& \rho_{0} \frac{\partial \boldsymbol{U}_{1}}{\partial t}=-\nabla p_{1}+\rho_{1} \boldsymbol{g}+\left(\boldsymbol{U}_{1} \times \boldsymbol{\omega}\right)+\boldsymbol{Q}_{1} \\
& \frac{\partial \rho_{1}}{\partial t}+\nabla \cdot\left(\rho_{0} \boldsymbol{U}_{1}\right)=0,
\end{aligned}
$$


where $\boldsymbol{Q}_{1}$ given in the appendix (see Equation (40)) We now appeal to the fact that, for many situations of interest in ICF (inertial-confinement fusion), unstable flow occurs at velocities much smaller than the local sound speed. This has the effect that accelerations in the flow are not strong enough to change the density of a fluid element significantly, so the fluid moves without compressing or expanding. In such a situation we call the flow incompressible. Provided that we are well away from shock waves or centers of convergence, the assumption of incompressible flow is often valid. To say that fluid elements move without changing density is to say that the Lagrangian total derivative of density is zero, that

$$
\frac{\mathrm{d} \rho}{\mathrm{d} t}=\left(\frac{\partial}{\partial t}+\boldsymbol{U} \cdot \nabla\right) \rho=0,
$$

We also linearize this equation, where the first-order quantities, becomes

$$
\frac{\partial \rho_{1}}{\partial t}+\left(\boldsymbol{U}_{1} \cdot \nabla\right) \rho_{0}=0 .
$$

Comparing this equation to Equation (5), which we can rewrite in expanded form as

$$
\frac{\partial \rho_{1}}{\partial t}+\left(\boldsymbol{U}_{1} \cdot \nabla\right) \rho_{0}+\rho_{0} \nabla \cdot \boldsymbol{U}_{1}=0
$$

we see that subtracting Equation (6) from Equation (7) yields

$$
\nabla \cdot \boldsymbol{U}_{1}=0 .
$$

This is a consequence of the assumption of incomepressible flow. We can use either Equation (6) or Equation (8) to replace the linearized continuity equation Equation (4) under this assumption. One can seen that the set of Equations (3), (6) and (8) is complete for describing the quantum effects on the Rayleigh-Taylor instability of incompressible rotating plasma. Now, where $\boldsymbol{U}_{1}=\left(u_{x 1}, u_{y 1}, u_{z 1}\right), \quad g=(0,0,-g)$ and $\boldsymbol{Q}_{1}=\left(Q_{x 1}, Q_{y 1}, Q_{z 1}\right)$. The fluid is arranged in horizontal strata, then $\rho_{0}$ is a function of the vertical coordinate only $\left(\rho_{0}=\rho_{0}(z)\right)$, and $p_{0}=p_{0}(z)$. So, the system Equations (3), (6) and (8) become

$$
\begin{gathered}
\rho_{0} \frac{\partial u_{x 1}}{\partial t}=-\frac{\partial p_{1}}{\partial x}-\rho_{0}\left(\omega_{z} u_{y 1}-\omega_{z} u_{z 1}\right)+Q_{x 1}, \\
\rho_{0} \frac{\partial u_{y 1}}{\partial t}=-\frac{\partial p_{1}}{\partial y}+\rho_{0} \omega_{z} u_{x 1}+Q_{y 1}, \\
\rho_{0} \frac{\partial u_{z 1}}{\partial t}=-\frac{\partial p_{1}}{\partial z}-\rho_{1} g-\rho_{0} \omega_{y} u_{x 1}+Q_{z 1}, \\
\frac{\partial \rho_{1}}{\partial t}+u_{z 1} \frac{\mathrm{d} \rho_{0}(z)}{\mathrm{d} z}=0, \\
\frac{\partial u_{x 1}}{\partial x}+\frac{\partial u_{y 1}}{\partial y}+\frac{\partial u_{z 1}}{\partial z}=0,
\end{gathered}
$$

where $Q_{x 1}, Q_{y 1}$ and $Q_{z 1}$ given in the appendix (see Equations (41)-(43)) Now, the perturbed quantifies are assumed to be proportional to

$$
f_{1}(x, y, z, t)=f(z) \exp \left(\mathrm{i}\left(k_{x} x+k_{y} y-\omega t\right)\right),
$$

where $k_{x}, k_{y}$ are the perpendicular and parallel component of the wave vector $k$ and $\omega$ (may be com$\left.\operatorname{plex}\left(\omega=\omega_{r}+\mathrm{i} \gamma\right)\right)$ is the frequency of perturbations. Inserting expression (14) in the system of Equations (9)(13), we have

$$
\begin{aligned}
& -\mathrm{i} \omega \rho_{0} u_{x 1}=-\mathrm{i} k_{x} p_{1}-\rho_{0}\left(\omega_{z} u_{y 1}-\omega_{z} u_{z 1}\right)+\bar{Q}_{x 1}, \\
& -\mathrm{i} \omega \rho_{0} u_{y 1}=-\mathrm{i} k_{y} p_{1}+\rho_{0} \omega_{z} u_{x 1}+\bar{Q}_{y 1}, \\
& -\mathrm{i} \omega \rho_{0} u_{z 1}=-\frac{\partial p_{1}}{\partial z}-\rho_{1} g-\rho_{0} \omega_{y} u_{x 1}+\bar{Q}_{z 1}, \\
& -\mathrm{i} \omega \rho_{1}+\left(\frac{\mathrm{d} \rho_{0}(z)}{\mathrm{d} z}\right) u_{z 1}=0, \\
& \mathrm{i} k_{x} u_{x 1}+\mathrm{i} k_{y} u_{y 1}+\frac{\partial u_{z 1}}{\partial z}=0,
\end{aligned}
$$

where $\boldsymbol{Q}_{x 1}, \boldsymbol{Q}_{y 1}$ and $\boldsymbol{Q}_{z 1}$ given in the appendix (see Equations (44)-(46)) Eliminating some variables from the system of Equations (14)-(19) we get a differential equation in $u_{z 1}$

$$
\begin{aligned}
& {\left[\rho_{0}\left(\omega^{2}-\omega_{z}^{2}\right)+k^{2} A\right] \frac{\mathrm{d}^{2} u_{z 1}}{\mathrm{~d} z^{2}}+\left[\left(\omega^{2}-\omega_{z}^{2}\right)\left(\frac{\mathrm{d} \rho_{0}}{\mathrm{~d} z}+\frac{\mathrm{i} k_{x} \omega_{y} \rho_{0}}{\frac{k_{x}}{k_{y}} \omega_{z}+\mathrm{i} \omega}\right)+\rho_{0} \omega \omega_{y}\left(\frac{\omega_{z}}{\mathrm{i} \omega} k_{y}-k_{x}\right)+\frac{\omega \omega_{y} \omega_{z} k^{2} \rho_{0}}{k_{y}\left(\frac{k_{x}}{k_{y}} \omega_{z}+\mathrm{i} \omega\right)}+k^{2} B\right] \frac{\mathrm{d} u_{z 1}}{\mathrm{~d} z}} \\
& +\left[-\rho_{0} k^{2} \omega^{2}+\omega \omega_{y}\left(\frac{\omega_{z}}{\mathrm{i} \omega} k_{y}-k_{x}\right)\left(\frac{\mathrm{d} \rho_{0}}{\mathrm{~d} z}\right)+\frac{\mathrm{i} \omega \omega_{y}^{2} k_{x} \rho_{0}\left(\frac{\omega_{z}}{\mathrm{i} \omega} k_{y}-k_{x}\right)}{\left(\frac{k_{x}}{k_{y}} \omega_{z}+\mathrm{i} \omega\right)}+\frac{\mathrm{i} \omega_{y}^{2} k^{2} \rho_{0}}{\left(\frac{k_{x}}{k_{y}} \omega_{z}+\mathrm{i} \omega\right)}+k^{2}(C-g)\left(\frac{\mathrm{d} \rho_{0}}{\mathrm{~d} z}\right)\right] u_{z 1}=0,
\end{aligned}
$$




$$
\begin{aligned}
& A=-\frac{\hbar^{2}}{4 m_{e} m_{i}} \frac{1}{\rho_{0}}\left(\frac{\mathrm{d} \rho_{0}}{\mathrm{~d} z}\right)^{2}, \\
& B=\frac{\hbar^{2}}{4 m_{e} m_{i}} \frac{1}{\rho_{0}^{2}}\left(\frac{\mathrm{d} \rho_{0}}{\mathrm{~d} z}\right)^{2}\left\{\left(\frac{\mathrm{d} \rho_{0}}{\mathrm{~d} z}\right)^{2}-2 \rho_{0} \frac{\mathrm{d}^{2} \rho_{0}}{\mathrm{~d} z^{2}}\right\}, \\
& C=\frac{\hbar^{2}}{4 m_{e} m_{i}} \frac{k^{2}}{\rho_{0}}\left(\frac{\mathrm{d} \rho_{0}}{\mathrm{~d} z}\right)
\end{aligned}
$$

\section{A Continuously Stratified Plasma Layer}

In this section we will consider the case of incompressible continuously stratified plasma layer of thickness $h$ units confined between two rigid boundaries, in which the density is given by $\rho_{0}(z)=\rho_{0}(0) \exp \left(z / L_{D}\right)$ (see Figure 1), where $\rho_{0}(0)$ and $L_{D}$ are constants, then Equation (20) takes the form

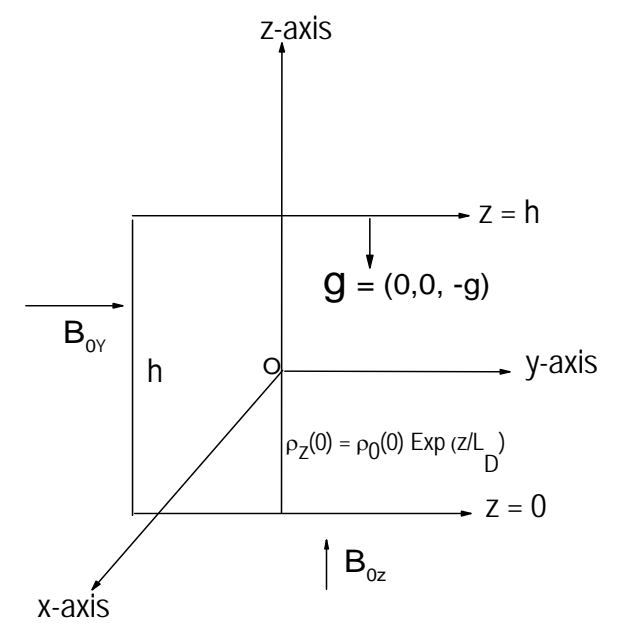

Figure 1. Schematic diagram of finite quantum plasma layer with external magnetic field.

$$
\begin{aligned}
& {\left[\omega^{2}-\omega_{z}^{2}-\omega_{q}^{2}\right] \frac{\mathrm{d}^{2} u_{z 1}}{\mathrm{dz}}+\left[\left(\omega^{2}-\omega_{z}^{2}\right)\left(\frac{1}{L_{D}}+\frac{\mathrm{i} k_{x} \omega_{y}}{\frac{k_{x}}{k_{y}} \omega_{z}+\mathrm{i} \omega}\right)+\omega \omega_{y}\left(\frac{\omega_{z}}{\mathrm{i} \omega} k_{y}-k_{x}\right)+\frac{\omega \omega_{y} \omega_{z} k^{2}}{k_{y}\left(\frac{k_{x}}{k_{y}} \omega_{z}+\mathrm{i} \omega\right)}-\frac{\omega_{q}^{2}}{L_{D}}\right] \frac{\mathrm{d} u_{z 1}}{\mathrm{~d} z}} \\
& +\left[-k^{2} \omega^{2}+\omega \omega_{y}\left(\frac{\omega_{z}}{\mathrm{i} \omega} k_{y}-k_{x}\right) \frac{1}{L_{D}}+\frac{\mathrm{i} \omega \omega_{y}^{2} k_{x}\left(\frac{\omega_{z}}{\mathrm{i} \omega} k_{y}-k_{x}\right)}{\left(\frac{k_{x}}{k_{y}} \omega_{z}+\mathrm{i} \omega\right)}+\frac{\mathrm{i} \omega \omega_{y}^{2} k^{2}}{\left(\frac{k_{x}}{k_{y}} \omega_{z}+\mathrm{i} \omega\right)}+k^{2}\left(\omega_{q}^{2}-\frac{g}{L_{D}}\right)\right] u_{z 1}=0,
\end{aligned}
$$

where $\omega_{q}^{2}=\frac{\hbar^{2} k^{2}}{4 L_{D}^{2} m_{e} m_{i}}$ represents quantum effect. Now, if we choose $u_{z 1}$ in the form $u_{z 1}=\sin \left(\frac{n \pi}{h} z\right) \exp (\lambda z)$ and by substituting in Equation (22), we have

$$
\begin{aligned}
& {\left[\omega^{2}-\omega_{z}^{2}-\omega_{q}^{2}\right]\left[\left(\lambda^{2}-\left(\frac{n \pi}{h}\right)^{2}\right) \sin \left(\frac{n \pi}{h} z\right)+\frac{2 n \pi}{h} \cos \left(\frac{n \pi}{h} z\right)\right]} \\
& +\left[\left(\omega^{2}-\omega_{z}^{2}\right)\left(\frac{1}{L_{D}}+\frac{i k_{x} \omega_{y}}{\frac{k_{x}}{k_{y}} \omega_{z}+\mathrm{i} \omega}\right)+\omega \omega_{y}\left(\frac{\omega_{z}}{\mathrm{i} \omega} k_{y}-k_{x}\right)+\frac{\omega \omega_{y} \omega_{z} k^{2}}{k_{y}\left(\frac{k_{x}}{k_{y}} \omega_{z}+\mathrm{i} \omega\right)}-\frac{\omega_{q}^{2}}{L_{D}}\right]\left[\lambda \sin \left(\frac{n \pi}{h} z\right)+\frac{n \pi}{h} \cos \left(\frac{n \pi}{h} z\right)\right] \\
& +\left[-k^{2} \omega^{2}+\omega \omega_{y}\left(\frac{\omega_{z}}{\mathrm{i} \omega} k_{y}-k_{x}\right) \frac{1}{L_{D}}+\frac{\mathrm{i} \omega \omega_{y}^{2} k_{x}\left(\frac{\omega_{z}}{\mathrm{i} \omega} k_{y}-k_{x}\right)}{\left(\frac{k_{x}}{k_{y}} \omega_{z}+\mathrm{i} \omega\right)}+\frac{\mathrm{i} \omega \omega_{y}^{2} k^{2}}{\left(\frac{k_{x}}{k_{y}} \omega_{z}+\mathrm{i} \omega\right)}+k^{2}\left(\omega_{q}^{2}-\frac{g}{L_{D}}\right)\right] \sin \left(\frac{n \pi}{h} z\right)=0
\end{aligned}
$$

Then coefficients both $\sin \left(\frac{n \pi}{h} z\right)$ and $\cos \left(\frac{n \pi}{h} z\right)$, respectively, are given by: 


$$
\begin{aligned}
& {\left[\lambda^{2}-\left(\frac{n \pi}{h}\right)^{2}\right]\left[\omega^{2}-\omega_{z}^{2}-\omega_{q}^{2}\right]+\lambda\left[\left(\omega^{2}-\omega_{z}^{2}\right)\left(\frac{1}{L_{D}}+\frac{\mathrm{i} k_{x} \omega_{y}}{\frac{k_{x}}{k_{y}} \omega_{z}+\mathrm{i} \omega}\right)+\omega \omega_{y}\left(\frac{\omega_{z}}{\mathrm{i} \omega} k_{y}-k_{x}\right)+\frac{\omega \omega_{y} \omega_{z} k^{2}}{k_{y}\left(\frac{k_{x}}{k_{y}} \omega_{z}+\mathrm{i} \omega\right)}-\frac{\omega_{q}^{2}}{L_{D}}\right]} \\
& +\left[-k^{2} \omega^{2}+\omega \omega_{y}\left(\frac{\omega_{z}}{\mathrm{i} \omega} k_{y}-k_{x}\right) \frac{1}{L_{D}}+\frac{\mathrm{i} \omega \omega_{y}^{2} k_{x}\left(\frac{\omega_{z}}{\mathrm{i} \omega} k_{y}-k_{x}\right)}{\left(\frac{k_{x}}{k_{y}} \omega_{z}+\mathrm{i} \omega\right)}+\frac{\mathrm{i} \omega \omega_{y}^{2} k^{2}}{\left(\frac{k_{x}}{k_{y}} \omega_{z}+\mathrm{i} \omega\right)}+k^{2}\left(\omega_{q}^{2}-\frac{g}{L_{D}}\right)\right]=0 \\
& 2 \lambda\left[\omega^{2}-\omega_{z}^{2}-\omega_{q}^{2}\right]+\left[\left(\omega^{2}-\omega_{z}^{2}\right)\left(\frac{1}{L_{D}}+\frac{\mathrm{i} k_{x} \omega_{y}}{\frac{k_{x}}{k_{y}} \omega_{z}+\mathrm{i} \omega}\right)+\omega \omega_{y}\left(\frac{\omega_{z}}{\mathrm{i} \omega} k_{y}-k_{x}\right)+\frac{\omega \omega_{y} \omega_{z} k^{2}}{k_{y}\left(\frac{k_{x}}{k_{y}} \omega_{z}+\mathrm{i} \omega\right)}-\frac{\omega_{q}^{2}}{L_{D}}\right]=0
\end{aligned}
$$

Now, where $\omega=\omega_{r}+\mathrm{i} \gamma$ and in the case of unstable $\omega=\mathrm{i} \gamma \quad\left(\omega_{r}^{*}=0\right.$, stable oscillations), then above equastratification the frequency should be replaced by the tions may be given by

$$
\begin{aligned}
& -\left[\lambda^{2}-\left(\frac{n \pi}{h}\right)^{2}\right]\left[\gamma^{2}+\omega_{z}^{2}+\omega_{q}^{2}\right]+\lambda\left[-\left(\gamma^{2}+\omega_{z}^{2}\right)\left(\frac{1}{L_{D}}+\frac{\mathrm{i} k_{x} \omega_{y}}{\frac{k_{x}}{k_{y}} \omega_{z}-\gamma}\right)-\mathrm{i} \gamma \omega_{y}\left(\frac{\omega_{z}}{\gamma} k_{y}+k_{x}\right)+\frac{\mathrm{i} \gamma \omega_{y} \omega_{z} k^{2}}{k_{y}\left(\frac{k_{x}}{k_{y}} \omega_{z}-\gamma\right)}-\frac{\omega_{q}^{2}}{L_{D}}\right] \\
& +\left[k^{2} \gamma^{2}-\mathrm{i} \gamma \omega_{y}\left(\frac{\omega_{z}}{\gamma} k_{y}+k_{x}\right) \frac{1}{L_{D}}+\frac{\gamma \omega_{y}^{2} k_{x}\left(\frac{\omega_{z}}{\gamma} k_{y}+k_{x}\right)}{\left(\frac{k_{x}}{k_{y}} \omega_{z}-\gamma\right)}-\frac{\gamma \omega_{y}^{2} k^{2}}{\left(\frac{k_{x}}{k_{y}} \omega_{z}-\gamma\right)}+k^{2}\left(\omega_{q}^{2}-\frac{g}{L_{D}}\right)\right]=0 \\
& -2 \lambda\left[\gamma^{2}+\omega_{z}^{2}+\omega_{q}^{2}\right]+\left[-\left(\gamma^{2}+\omega_{z}^{2}\right)\left(\frac{1}{L_{D}}+\frac{\mathrm{i} k_{x} \omega_{y}}{\frac{k_{x}}{k_{y}} \omega_{z}-\gamma}\right)-\mathrm{i} \gamma \omega_{y}\left(\frac{\omega_{z}}{\gamma} k_{y}+k_{x}\right)+\frac{\mathrm{i} \gamma \omega_{y} \omega_{z} k^{2}}{k_{y}\left(\frac{k_{x}}{k_{y}} \omega_{z}-\gamma\right)}-\frac{\omega_{q}^{2}}{L_{D}}\right]=0 .
\end{aligned}
$$

The real parts of the above two equations, respectively, are as

$$
\begin{aligned}
& {\left[\lambda^{2}-\left(\frac{n \pi}{h}\right)^{2}+\frac{\lambda}{L_{D}}\right]\left[\gamma^{2}+\omega_{z}^{2}+\omega_{q}^{2}\right]-\left[k^{2} \gamma^{2}+\frac{\gamma \omega_{y}^{2}\left\{k_{x}\left(\frac{\omega_{z}}{\gamma} k_{y}+k_{x}\right)-k^{2}\right\}}{\left(\frac{k_{x}}{k_{y}} \omega_{z}-\gamma\right)}+k^{2}\left(\omega_{q}^{2}-\frac{g}{L_{D}}\right)\right]=0,} \\
& \lambda=-\frac{1}{2 L_{D}} .
\end{aligned}
$$

Substituting from Equation (29) into Equation (28), the dispersion relation becomes 


$$
\begin{aligned}
& {\left[\left(\frac{n \pi}{h}\right)^{2}+\frac{1}{4 L_{D}^{2}}+k^{2}\right] \gamma^{2}+\left[\left(\frac{n \pi}{h}\right)^{2}+\frac{1}{4 L_{D}^{2}}+k^{2}\right] \omega_{q}^{2}} \\
& +\left[\left(\frac{n \pi}{h}\right)^{2}+\frac{1}{4 L_{D}^{2}}\right] \omega_{z}^{2}+\left[\frac{\gamma \omega_{y}^{2}\left\{k_{x}\left(\frac{\omega_{z}}{\gamma} k_{y}+k_{x}\right)-k^{2}\right\}}{\left(\frac{k_{x}}{k_{y}} \omega_{z}-\gamma\right)}-g k^{2} \frac{1}{L_{D}}\right]=0 .
\end{aligned}
$$

Now, if we define $\left(\boldsymbol{k} \times \boldsymbol{\omega}_{y}\right)_{\boldsymbol{e}_{z}}=k_{x} \omega_{y},\left(\boldsymbol{k} \cdot \boldsymbol{\omega}_{y}\right)=k_{y} \omega_{y} . \quad$ and Then

$$
\begin{aligned}
& \frac{k_{x}}{k_{y}}=\frac{\left(\boldsymbol{k} \times \boldsymbol{\omega}_{y}\right)_{\boldsymbol{e}_{z}}}{\left(\boldsymbol{k} \cdot \boldsymbol{\omega}_{y}\right)}, \omega_{y}^{2} k_{x} k_{y}=\left(\boldsymbol{k} \times \boldsymbol{\omega}_{y}\right)_{\boldsymbol{e}_{z}}\left(\boldsymbol{k} \cdot \boldsymbol{\omega}_{y}\right) \omega_{y}^{2}\left(k_{x}^{2}-k^{2}\right)= \\
& {\left[\left(\frac{n \pi}{h}\right)^{2}+\frac{1}{4 L_{D}^{2}}+k^{2}\right] \gamma^{2}+\left[\left(\frac{n \pi}{h}\right)^{2}+\frac{1}{4 L_{D}^{2}}+k^{2}\right] \omega_{q}^{2}+\left[\left(\frac{n \pi}{h}\right)^{2}+\frac{1}{4 L_{D}^{2}}\right] \omega_{z}^{2} } \\
&+\left[\frac{\omega_{z}\left(\boldsymbol{k} \times \omega_{y}\right)_{\boldsymbol{e}_{z}}\left(\boldsymbol{k} \cdot \boldsymbol{\omega}_{y}\right)-\gamma\left(\boldsymbol{k} \cdot \boldsymbol{\omega}_{y}\right)^{2}}{\left(\boldsymbol{k} \times \boldsymbol{\omega}_{y}\right)_{\boldsymbol{e}_{z}}}-\gamma k^{2} \frac{1}{L_{D}}\right]=0 \\
&\left.\omega_{z} \frac{\left(\boldsymbol{k} \cdot \omega_{y}\right)}{n}\right]
\end{aligned}
$$

\section{Special Cases}

To discuss the different roles of the parameter's problem, the next special cases are considered from Equation (31). In the next steps, we will define the dimensionless quantities

$$
\begin{aligned}
& \gamma^{*^{2}}=\frac{\gamma^{2}}{\omega_{p e}^{2}}, \omega_{z}^{*^{2}}=\frac{\omega_{z}^{2}}{\omega_{p e}^{2}}, \omega_{y}^{*^{2}}=\frac{\omega_{y}^{2}}{\omega_{p e}^{2}}, \omega_{q}^{*^{2}}=\frac{\hbar^{2}}{4 L_{D}^{4} m_{e} m_{i} \omega_{p e}^{2}}, \lambda^{* 2}=\lambda^{2} L_{D}^{2}, h^{* 2}=\frac{h^{2}}{L_{D}^{2}}, \\
& k^{*^{2}}=k^{2} L_{D}^{2}, g^{*}=\frac{g}{\omega_{p e}^{2} L_{D}}, \omega_{p e}=\left(\frac{\rho_{0} e^{2}}{m_{e}^{2} \varepsilon_{0}}\right)^{1 / 2},
\end{aligned}
$$

1) In the case $\omega_{y}=\omega_{z}=0, \omega_{q}=0$, the normalized growth rate from Equation (32) given by

$$
\gamma_{\text {classical }}^{*}=\sqrt{\frac{4 g^{*} k^{*^{2}} h^{*^{2}}}{h^{*^{2}}+4 k^{*^{2}} h^{*^{2}}+4 n^{2} \pi^{2}}} .
$$

This case is considered by Goldston and Rutherford (see Ref. [7]), which represents an exponentially growing perturbation (instability case).

2) In the case $\omega_{y}=\omega_{z}=0, \omega_{q} \neq 0$, the normalized growth rate from Equation (31) given by

$$
\begin{aligned}
& \gamma_{\text {quantum }}^{*}=\gamma^{*}\left(\omega_{q}\right) \\
& =\sqrt{\frac{4 g^{*} k^{*^{2}} h^{*^{2}}}{h^{*^{2}}+4 k^{*^{2}} h^{*^{2}}+4 n^{2} \pi^{2}}-k^{*^{2}} \omega_{q}^{*^{2}}},
\end{aligned}
$$

This case is studied in Refs. [23-29]. It is clarified that, the quantum term has stabilizing effect on RTI problem. This influence is obvious from Equations (33) and (34), where $\gamma_{\text {quantum }}^{*}<\gamma_{\text {classical }}^{*}$. In this case, the system increases as $k^{*}$ increases through the range $0<k^{*}<k_{\max }^{*}$, and when $k^{*}>k_{\max }^{*}$ it starts to decreases as $k^{*}$ increases, where

$$
k_{\max }^{*}=\sqrt{\frac{1}{\omega_{q}^{*}}\left(\sqrt{g^{*}}-1\right)\left(\frac{1}{4}+\left(\frac{n \pi}{h^{*}}\right)^{2}\right)} .
$$

At $k^{*}=k_{c}^{*}$ the system arrives to complete stability case, where

$$
k_{c}^{*}=\sqrt{\frac{g^{*}}{\omega_{q}^{*^{2}}}-\left(\frac{1}{4}+\left(\frac{n \pi}{h^{*}}\right)^{2}\right)}
$$

3) In the case $\omega_{y}=0, \omega_{z} \neq 0, \omega_{q} \neq 0$, also the normal- 
ized growth rate from Equation (31) given by

$$
\gamma^{*}\left(\omega_{z}, \omega_{q}\right)=\sqrt{\frac{4 g^{*} k^{*^{2}} h^{*^{2}}}{h^{*^{2}}+4 k^{*^{2}} h^{*^{2}}+4 n^{2} \pi^{2}}-\left\{k^{*^{2}} \omega_{q}^{*^{2}}+\frac{h^{*^{2}}+4 n^{2} \pi^{2}}{h^{*^{2}}+4 k^{*^{2}} h^{*^{2}}+4 n^{2} \pi^{2}} \omega_{z}^{*^{2}}\right\}},
$$

See Equation (21) ref. [25]. From the system of Equation (33)-(35), it is clear that, the external vertical magnetic field in the presence of quantum correction plays more stabilizing role on the considerable model.

4) In the case $\omega_{y} \neq 0, \omega_{z}=0, \omega_{q} \neq 0$, from Equation (31) the normalized growth rate given from:

$$
\left[\left(\frac{n \pi}{h}\right)^{2}+\frac{1}{4 L_{D}^{2}}+k^{2}\right] \gamma^{2}+\left[\left(\frac{n \pi}{h}\right)^{2}+\frac{1}{4 L_{D}^{2}}+k^{2}\right] \omega_{q}^{2}+\left[\left(\boldsymbol{k} \cdot \boldsymbol{\omega}_{y}\right)^{2}-g k^{2} \frac{1}{L_{D}}\right]=0,
$$

This equation corresponds both the special cases in Equations (16) and (17) (see Hoshoudy [26]).

5) For the general case $\omega_{y} \neq 0, \omega_{z} \neq 0, \omega_{q} \neq 0$ we consider the next cases: (a) At $\boldsymbol{k} \perp \boldsymbol{\omega}_{y}=0$, we have that $=\left(\boldsymbol{k} \times \boldsymbol{\omega}_{y}\right)_{\boldsymbol{e}}=k \omega_{y}$ and $\boldsymbol{k} \cdot \boldsymbol{\omega}_{y}=0$. So, the normalized growth rate from Equation (31) given by

$$
\gamma^{*}\left(\omega_{y}, \omega_{z}, \omega_{q}\right)=\sqrt{\frac{4 g^{*} k^{*^{2}} h^{*^{2}}}{h^{*^{2}}+4 k^{*^{2}} h^{*^{2}}+4 n^{2} \pi^{2}}-\left\{k^{*^{2}} \omega_{q}^{*^{2}}+\frac{h^{*^{2}}+4 n^{2} \pi^{2}}{h^{*^{2}}+4 k^{*^{2}} h^{*^{2}}+4 n^{2} \pi^{2}} \omega_{z}^{*^{2}}\right\}} .
$$

This case corresponds the case of $\omega_{y}=0$, that is above cleared in Equation (35). Which implies that, the role of external horizontal magnetic vanishes when this force is vertical to the wave number. (b) At $\boldsymbol{k} \| \boldsymbol{\omega}_{y}$, we have that $\left(\boldsymbol{k} \times \boldsymbol{\omega}_{y}\right)_{\boldsymbol{e}}=0$ and $\boldsymbol{k} \cdot \boldsymbol{\omega}_{y}=-k \omega$. So, the normalized growth rate from Equation (31) given by

$$
\gamma_{\left(\omega_{y}, \omega_{z}, \omega_{q}\right)}^{*}=\sqrt{\frac{4 g^{*} k^{*^{2}} h^{*^{2}}}{h^{*^{2}}+4 k^{*^{2}} h^{*^{2}}+4 n^{2} \pi^{2}}-\left\{k^{*^{2}} \omega_{q}^{*^{2}}+\frac{1}{h^{*^{2}}+4 k^{*^{2}} h^{*^{2}}+4 n^{2} \pi^{2}} \times\left[\left(h^{*^{2}}+4 n^{2} \pi^{2}\right) \omega_{z}^{*^{2}}+4 h^{*^{2}} k^{*^{2}} \omega_{y}^{*^{2}}\right]\right\}},
$$

where, it clears that, the magnitudes of growth rate in the presence of external horizontal and vertical magnetic field beside the quantum effect case are lees than their counterpart in the absence of either them $\left(\gamma_{\left(\omega_{y}, \omega_{z}, \omega_{q}\right)}^{*}<\gamma_{\text {classical }}^{*}\right)$ and this magnitudes decreases with the ${ }^{\prime}$ increasing of the external horizontal and vertical magnetic field and the quantum effect. Also, in this case the complete stability happens at

$$
\begin{aligned}
k_{c}^{* 2} & =\frac{1}{2}\left[\frac{1}{4 h^{*^{2}} \omega_{q}^{*^{2}}}\left[4 h^{*^{2}}\left(g^{*}-\omega_{y}^{*^{2}}\right)-\omega_{q}^{*^{2}}\left(h^{*^{2}}++4 n^{2} \pi^{2}\right)\right]\right. \\
& \left. \pm \sqrt{\left\{\frac{1}{4 h^{* 2} \omega_{q}^{* 2}}\left[4 h^{*^{2}}\left(g^{*}-\omega_{y}^{*^{2}}\right)-\omega_{q}^{*^{2}}\left(h^{*^{2}}++4 n^{2} \pi^{2}\right)\right]\right\}^{2}-\frac{\omega_{z}^{*^{2}}}{h^{*^{2}} \omega_{q}^{*^{2}}}\left(h^{*^{2}}+4 n^{2} \pi^{2}\right)}\right]
\end{aligned}
$$

This equation indicates that, the critical point $\left(k_{c}^{*^{2}}\right)$ of stability is affected in presence of $\omega_{y}^{*}$ and $\omega_{z}^{*}$, which decreases with the increasing of both them. Last but not least, the presence of external horizontal and vertical magnetic field beside the quantum effect will bring about more stability on the considerable model, when the vector of the waver number parallels to the vector of the external horizontal magnetic. The same formula (i.e. Equation (38)) holds if both $\boldsymbol{k}$ and $\boldsymbol{\omega}_{y}$ are in the same direction, where $\left(\boldsymbol{k} \times \boldsymbol{\omega}_{y}\right)_{\boldsymbol{e}_{z}}=0$, and $\boldsymbol{k} \cdot \boldsymbol{\omega}_{y}=k \omega$.

These notes are presented in Figure 2, where the square normalized growth rate $\gamma^{* 2}$ is plotted against the square normalized wave number $k^{*^{2}}$. In this figure it notes the stabilizing role that plays the parameters $\omega_{q}^{*}, \omega_{y}^{*}$ and $\omega_{z}^{*}$ on the Rayleigh-Taylor instability, where the square normalized growth rate in the presence of this parameters or either them is less than that at the classical case. The quantum term has critical strength to suppress the instability completely, while both the external horizontal or vertical magnetic field has no like this strength. This implies that, the quantum term who that do the critical point $k_{c}^{*^{2}}$ for the stability that is affected by presence of both 


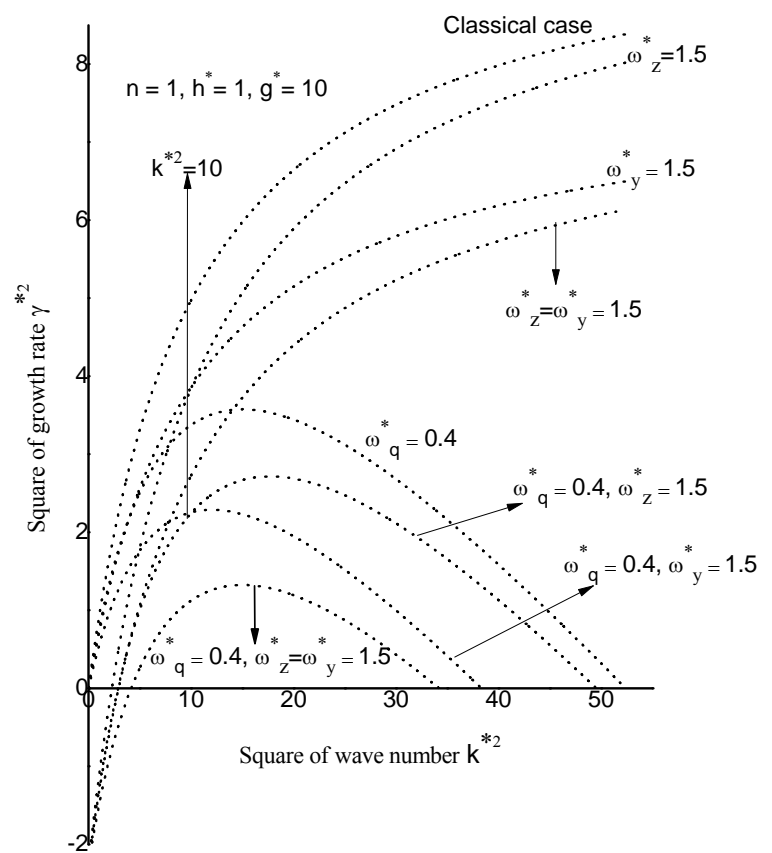

Figure 2. The square normalized growth rate $\gamma^{*^{2}}$ against the square normalized wave number $k^{*^{2}}$ at $n=1, h^{*}=1, g^{*}$ $=10$ for a different values of $\omega_{q}^{*}, \omega_{y}^{*}$ and $\omega_{z}^{*}$.

$\omega_{y}^{*}$ and $\omega_{z}^{*}$, where at $\omega_{q}^{*}=0.4$ the square critical point is $k_{c}^{*^{2}}=52.4$, while in the presence of external magnetic filed it given as $\omega_{y}^{*}=1.5\left(k_{c}^{*^{2}}=38.4\right), \omega_{z}^{*}=1.5$

$\left(k_{c}^{*^{2}}=49.5\right)$ and $\omega_{y}^{*}=\omega_{z}^{*}=1.5 \quad\left(k_{c}^{*^{2}}=34.2\right)$. Also, from this figure and for the same values of both external horizontal and vertical magnetic field, it is clear that, the stabilizing role that plays the external vertical magnetic field at $k^{*^{2}}<10$ is greater than that plays the external horizontal magnetic field in the presence of or absence of quantum term, while this role changes at $k^{*^{2}}>10$, where the role of external horizontal magnetic field will be greatest and this role rises in the square of critical point of stability $\left(k_{c}^{*^{2}}=38.4\right.$ at $\left.\omega_{y}^{*}=1.5\right)$. The external vertical magnetic field has critical strength to suppress the instability completely at small values of normalized wave number and in this stage the system capitulates to the external vertical magnetic field effect.

\section{Conclusion}

The effect of quantum mechanism with external horizontal and vertical magnetic field on the Rayleigh-Taylor instability of stratified incompressible rotating plasmas layer is studied. We can summarize the results as follows:

1) The role of external horizontal magnetic field $\omega_{y}$ will be vanish at $\boldsymbol{k} \perp \boldsymbol{\omega}_{y}=0$.
2) The normalized growth rate takes the same magnitudes at $\boldsymbol{k}|| \boldsymbol{\omega}_{y}$ and at $\boldsymbol{k}, \boldsymbol{\omega}_{y}$ are in the same direction.

3) The critical point for stability and that happens in the presence of quantum term it is affected by the presence of both external horizontal and vertical magnetic field.

4) For the same values of both external horizontal and vertical magnetic field, The stabilizing role of external vertical magnetic field will be greater than his counterpart of external horizontal magnetic field, if

$k^{*^{2}}<\frac{h^{*^{2}}+4 n^{2} \pi^{2}}{4 h^{*^{2}}} \quad$ (see Equation (38)), while this role will be change, if $k^{*^{2}}>\frac{h^{*^{2}}+4 n^{2} \pi^{2}}{4 h^{*^{2}}}$.

5) The quantum effect dissipates the energy of any disturbance more than that has been of both external horizontal and vertical magnetic field. In other words, the role of both external horizontal and vertical magnetic field, it is helping the quantum effect to finding more stability on RTI problem, while the quantum effect plays the fundamental role to generate the complete stability.

6) The stabilizing effect that happens in the presence of quantum mechanism and of both external horizontal and vertical magnetic field may be physically interpreted as apart of the kinetic energy of the waves has been absorbed, which leads to damping in the frequency of the waves. Finally, the considerable model is more stability then these are considered in Refs. [24-27]). This divergence ascribes to the stabilizing role that plays the presence of both external horizontal and vertical magnetic field on RTI problem, where the presence of external horizontal and vertical magnetic dissipates the energy of any disturbance and thereby there is more stability.

\section{REFERENCES}

[1] L. C. Gardner, "The Quantum Hydrodynamic Model for Semiconductor Devices," SIAM Journal on Applied Mathematics, Vol. 54, No. 2, 1994, pp. 409-427. doi:10.1137/S0036139992240425

[2] F. Haas "A Magnetohydrodynamic Model for Quantum Plasmas," Physics of Plasmas, Vol. 12, No. 6, 2005, Article ID: 062117, p 9. doi:10.1063/1.1939947

[3] L. Rayleigh, "Investigation of the Character of the Equilibrium of an Incompressible Heavy Fluid of Variable Density," Proceedings London Mathematical Society, Vol. 14 , No. 1,1882 , pp. 170-177. doi:10.1112/plms/s1-14.1.170

[4] G. I. Taylor, "The Instability of Liquid Surfaces when Accelerated in a Direction Perpendicular to Their Planes," Proceedings of the Royal Society of London. Series A, Vol. 201, No. 1065, 1950, pp. 192-196. doi:10.1098/rspa.1950.0052 
[5] K. Bhimsen Shivamoggi, "Rayleigh-Taylor Instability of Compressible Plasma in a Vertical Magnetic Field," Astrophysics and Space Science, Vol. 79, No. 1, 1981, pp. 3-9. doi:10.1007/BF00655900

[6] S. Liberatore, S. Jaoue, E. Tabakhoff and B. Canaud, "Compressible Magnetic Rayleigh-Taylor Instability in Stratified Plasmas: Comparison of Analytical and $\mathrm{Nu}-$ merical Results in the Linear Regime," Physics of Plasmas, Vol. 16, No. 4, 2009, Article ID: 044502, p 4. doi:10.1063/1.3109664

[7] R. J. Goldston and P. H. Rutherford, "Introduction to Plasma Physics Institute of Physics," Taylor \& Francis, London, 1997. doi:10.1201/9781439822074

[8] Z. Wu, W. Zhang, D. Li and W. Yang, "Effect of Magnetic Field and Equilibrium Flow on Rayleigh-Taylor Instability," Chinese Physics Letters, Vol. 21, No. 10, 2004, pp. 2001-2004. doi:10.1088/0256-307X/21/10/038

[9] P. A. Markowic, C. A. Ringhofer and C. Schmeiser, "Semiconductor Equations," Springer-Verlag, New York, 1990. doi:10.1007/978-3-7091-6961-2

[10] M. Opher, L. O. Silva, D. E. Dauger, V. K. Decyk and J. M. Dawson, "Nuclear Reaction Rates and Energy in Stellar Plasmas: The Effect of Highly Damped Modes," Physics of Plasmas, Vol. 8, No. 5, 2001, pp. 2454-2460. doi:10.1063/1.1362533

[11] Y. D. Jung, "Quantum-Mechanical Effects on ElectronElectron Scattering in Dense High-Temperature Plasmas," Physics of Plasmas, Vol. 8, 2001, p. 83842.

[12] D. Kremp, Th. Bornath, M. Bonitz and M. Schlanges, "Quantum Kinetic Theory of Plasmas in Strong Laser Fields," Physical Review E, Vol. 60, No. 4, 1999, pp. 4725-4732. doi:10.1103/PhysRevE.60.4725

[13] M. Leontovich, "On a Method for Solving the Problem of Electromagnetic Wave Propagation along the Earth Surface," Lzv. Akad. Nauk SSSR. Ser. Fiz, Vol. 8, 1994, pp. 16-22.

[14] G. Agrawal, "Nonlinear Fiber Optics," Academic Press, San Diego, 1995.

[15] G. Manfredi and F. Haas, "Self-Consistent Fluid Model for a Quantum Electron Gas," Physical Review B, Vol. 64, No. 7, 2001, Article ID: 075316, p 7. doi:10.1103/PhysRevB.64.075316

[16] G. Manfredi, "How to Model Quantum Plasmas," Fields Institute Communications Series, Vol. 46, 2005, pp. 263287.

[17] G. Gardner, "The Quantum Hydrodynamic Model for Semiconductor Devices," SIAM Journal on Applied Mathematics, Vol. 54, No. 2, 1994, pp. 409-427. doi:10.1137/S0036139992240425

[18] F.Haas, G. Manfredi and M. Feix, "Multistream Model for Quantum Plasmas," Physical Review E, Vol. 62, No. 2,
2000, pp. 2763-2772. doi:10.1103/PhysRevE.62.2763

[19] B. Eliasson and P. K. Shukla, "Dispersion Properties of Electrostatic Oscillations in Quantum Plasmas," Journal of Plasma Physics, Vol. 76, No. 1, 2010, pp. 7-17. doi:10.1017/S0022377809990316

[20] J. H. Jeans, "Astronomy and Cosmogony," Cambridge University Press, Cambridge, 2009.

[21] B. Vitaly, M. Marklund and M. Modestov, "The Rayleigh-Taylor Instability and Internal Waves in Quantum Plasmas," Physics Letters A, Vol. 372, No. 17, 2008, pp. 3042-3045. doi:10.1016/j.physleta.2007.12.065

[22] J. T. Cao, H. J. Ren, Z. W. Wu and P. K. Chu, "Quantum Effects on Rayleigh-Taylor Instability in Magnetized Plasma," Physics of Plasmas, Vol. 15, No. 1, 2008, Article ID: 012110 . doi:10.1063/1.2833588

[23] G. A. Hoshoudy, "Quantum Effects on Rayleigh-Taylor Instability of Incompressible Plasma in a Vertical Magnetic Field," Chinese Physics Letters, Vol. 27, No. 12, 2010, Article ID: 125201. doi:10.1088/0256-307X/27/12/125201

[24] G. A. Hoshoudy, "Rayleigh-Taylor Instability in Quantum Magnetized Viscous Plasma," Plasma Physics Reports, Vol. 37, No. 9, 2011, pp. 775-784. doi:10.1134/S1063780X11080046

[25] G. A. Hoshoudy, "Quantum Effects on Rayleigh-Taylor Instability in a Vertical Inhomogeneous Rotating Plasma," Physics of Plasmas, Vol. 16, No. 2, 2009, Article ID: 024501, p 4. doi:10.1063/1.3080202

[26] G. A. Hoshoudy, "Quantum Effects on Rayleigh-Taylor Instability in a Horizontal Inhomogeneous Rotating Plasma," Physics of Plasmas, Vol. 16, No. 6, 2009, Article ID: $064501, \mathrm{p} 4$.

[27] M. Modestov, V. Bychkov and M. Marklund, "The Rayleigh-Taylor Instability in Quantum Magnetized Plasma with Para- and Ferromagnetic Properties," Physics of Plasmas, Vol. 16, No. 3, 2009, Article ID: 032106, p 12. doi:10.1063/1.3085796

[28] G. A. Hoshoudy, "Quantum Effects on the RayleighTaylor Instability of Stratified Fluid/Plasma through Porous Media," Physics Letters A, Vol. 373, No. 30, 2009, pp. 2560-2567.

[29] G. A. Hoshoudy, "Quantum Effects on the RayleighTaylor Instability of Stratified Fluid/Plasma through Brinkman Porous Media," Journal of Porous Media, Vol. 15, No. 4, 2012, pp. 373-381. doi:10.1615/JPorMedia.v15.i4.50

[30] S. Ali, Z. Ahmed, M. Arshad Mirza and I. Ahmad, "Rayleigh-Taylor/Gravitational Instability in Dense Magnetoplasmas," Physics Letters A, Vol. 373, No. 33, 2009, pp. 2940-2946. doi:10.1016/i.physleta.2009.06.021 


\section{Appendix}

$$
\begin{aligned}
& \boldsymbol{Q}_{1}=\frac{\hbar^{2}}{2 m_{e} m_{i}}\left\{\frac{1}{2} \nabla\left(\nabla^{2} \rho_{1}\right)-\frac{1}{2 \rho_{0}} \nabla \rho_{1} \nabla^{2} \rho_{0}-\frac{1}{2 \rho_{0}} \nabla \rho_{0} \nabla^{2} \rho_{1}+\frac{\rho_{1}}{2 \rho_{0}^{2}} \nabla \rho_{0} \nabla^{2} \rho_{0}-\frac{1}{2 \rho_{0}} \nabla\left(\nabla \rho_{0} . \nabla \rho_{1}\right)\right. \\
& \left.+\frac{\rho_{1}}{4 \rho_{0}^{2}} \nabla\left(\nabla \rho_{0}\right)^{2}+\frac{1}{2 \rho_{0}^{2}}\left(\nabla \rho_{0}\right)^{2} \nabla \rho_{1}+\frac{1}{\rho_{0}^{2}}\left(\nabla \rho_{0} \cdot \nabla \rho_{1}\right) \nabla \rho_{0}-\frac{\rho_{1}}{\rho_{0}^{3}}\left(\nabla \rho_{0}\right)^{3}\right\} \\
& Q_{x 1}=\frac{\hbar^{2}}{2 m_{e} m_{i}} \frac{\partial}{\partial x}\left\{\frac{1}{2} \frac{\mathrm{d}^{2} \rho_{1}}{\mathrm{~d} z^{2}}-\frac{1}{2 \rho_{0}} \frac{\mathrm{d} \rho_{0}}{\mathrm{~d} z} \frac{\mathrm{d} \rho_{1}}{\mathrm{~d} z}+\left\{\frac{1}{2}\left(\frac{\partial^{2}}{\partial x^{2}}+\frac{\partial^{2}}{\partial y^{2}}\right)-\frac{1}{2 \rho_{0}} \frac{\mathrm{d}^{2} \rho_{0}}{\mathrm{~d} z^{2}} \frac{1}{2 \rho_{0}^{2}}\left(\frac{\mathrm{d} \rho_{0}}{\mathrm{~d} z}\right)^{2}\right\} \rho_{1}\right\}, \\
& Q_{y 1}=\frac{\hbar^{2}}{2 m_{e} m_{i}} \frac{\partial}{\partial y}\left\{\frac{1}{2} \frac{\mathrm{d}^{2} \rho_{1}}{\mathrm{~d} z^{2}}-\frac{1}{2 \rho_{0}} \frac{\mathrm{d} \rho_{0}}{\mathrm{~d} z} \frac{\mathrm{d} \rho_{1}}{\mathrm{~d} z}+\left\{\frac{1}{2}\left(\frac{\partial^{2}}{\partial x^{2}}+\frac{\partial^{2}}{\partial y^{2}}\right)-\frac{1}{2 \rho_{0}} \frac{\mathrm{d}^{2} \rho_{0}}{\mathrm{~d} z^{2}} \frac{1}{2 \rho_{0}^{2}}\left(\frac{\mathrm{d} \rho_{0}}{\mathrm{~d} z}\right)^{2}\right\} \rho_{1}\right\}, \\
& Q_{z 1}=\frac{\hbar^{2}}{2 m_{e} m_{i}}\left\{\frac{1}{2} \frac{\mathrm{d}^{3} \rho_{1}}{\mathrm{~d} z^{3}}-\frac{1}{\rho_{0}} \frac{\mathrm{d} \rho_{0}}{\mathrm{~d} z} \frac{\mathrm{d}^{2} \rho_{1}}{\mathrm{~d} z^{2}}+\left\{\frac{1}{2}\left(\frac{\partial^{2}}{\partial x^{2}}+\frac{\partial^{2}}{\partial y^{2}}\right)-\frac{1}{\rho_{0}} \frac{\mathrm{d}^{2} \rho_{0}}{\mathrm{~d} z^{2}}+\frac{3}{2 \rho_{0}^{2}}\left(\frac{\mathrm{d} \rho_{0}}{\mathrm{~d} z}\right)^{2}\right\} \frac{\mathrm{d} \rho_{1}}{\mathrm{~d} z}\right. \\
& \left.+\left\{-\frac{1}{2 \rho_{0}} \frac{\mathrm{d} \rho_{0}}{\mathrm{~d} z}\left(\frac{\partial^{2}}{\partial x^{2}}+\frac{\partial^{2}}{\partial y^{2}}\right)+\frac{1}{2 \rho_{0}^{2}} \frac{\mathrm{d} \rho_{0}}{\mathrm{~d} z} \frac{\mathrm{d}^{2} \rho_{0}}{\mathrm{~d} z^{2}}-\frac{1}{\rho_{0}^{3}}\left(\frac{\mathrm{d} \rho_{0}}{\mathrm{~d} z}\right)^{3}\right\} \rho_{1}\right\} \\
& \bar{Q}_{x 1}=\frac{\hbar^{2} k_{x}}{2 \varepsilon \omega m_{e} m_{i}}\left\{\frac{1}{2} \frac{\mathrm{d} \rho_{0}}{\mathrm{~d} z} \frac{\mathrm{d}^{2} u_{z 1}}{\mathrm{~d} z^{2}}+\left(\frac{\mathrm{d}^{2} \rho_{0}}{\mathrm{~d} z^{2}}-\frac{1}{2 \rho_{0}}\left(\frac{\mathrm{d}^{2} \rho_{0}}{\mathrm{~d} z^{2}}\right)^{2}\right) \frac{\mathrm{d} u_{z 1}}{\mathrm{~d} z}\right. \\
& \left.+\left(\frac{1}{2} \frac{\mathrm{d}^{3} \rho_{0}}{\mathrm{~d} z^{3}}-\frac{1}{\rho_{0}} \frac{\mathrm{d} \rho_{0}}{\mathrm{~d} z} \frac{\mathrm{d}^{2} \rho_{0}}{\mathrm{~d} z^{2}}-\frac{k^{2}}{2} \frac{\mathrm{d} \rho_{0}}{\mathrm{~d} z}+\frac{1}{2 \rho_{0}^{2}}\left(\frac{\mathrm{d} \rho_{0}}{\mathrm{~d} z}\right)^{3}\right) u_{z 1}\right\}^{\prime} \\
& \bar{Q}_{y 1}=\frac{k_{y}}{k_{x}} \bar{Q}_{x 1}, \\
& \bar{Q}_{z 1}=\frac{\hbar^{2}}{2 \mathrm{i} \varepsilon \omega m_{e} m_{i}}\left[\frac{1}{2} \frac{\mathrm{d} \rho_{0}}{\mathrm{~d} z} \frac{\mathrm{d}^{3} u_{z 1}}{\mathrm{dz} z^{3}}+\left(\frac{3}{2} \frac{\mathrm{d}^{2} \rho_{0}}{\mathrm{~d} z^{2}}-\frac{1}{\rho_{0}}\left(\frac{\mathrm{d}^{2} \rho_{0}}{\mathrm{dz})^{2}}\right)^{2}\right) \frac{\mathrm{d}^{2} u_{z 1}}{\mathrm{~d} z^{2}}\right. \\
& +\left(\frac{3}{2} \frac{\mathrm{d}^{3} \rho_{0}}{\mathrm{~d} z^{3}}-\frac{3}{\rho_{0}} \frac{\mathrm{d} \rho_{0}}{\mathrm{~d} z} \frac{\mathrm{d}^{2} \rho_{0}}{\mathrm{~d} z^{2}}-\frac{k^{2}}{2} \frac{\mathrm{d} \rho_{0}}{\mathrm{~d} z}+\frac{3}{2 \rho_{0}^{2}}\left(\frac{\mathrm{d} \rho_{0}}{\mathrm{~d} z}\right)^{3}\right) \frac{\mathrm{d} u_{z 1}}{\mathrm{~d} z} \\
& +\left(\frac{1}{2} \frac{\mathrm{d}^{4} \rho_{0}}{\mathrm{~d} z^{4}}-\frac{1}{\rho_{0}} \frac{\mathrm{d} \rho_{0}}{\mathrm{~d} z} \frac{\mathrm{d}^{3} \rho_{0}}{\mathrm{~d} z^{3}}-\frac{k^{2}}{2} \frac{\mathrm{d}^{2} \rho_{0}}{\mathrm{~d} z^{2}}-\frac{1}{\rho_{0}}\left(\frac{\mathrm{d}^{2} \rho_{0}}{\mathrm{~d} z^{2}}\right)^{2}+\right. \\
& \left.\left.\frac{5}{2 \rho_{0}^{2}}\left(\frac{\mathrm{d} \rho_{0}}{\mathrm{~d} z}\right)^{2} \frac{\mathrm{d}^{2} \rho_{0}}{\mathrm{~d} z^{2}}+\frac{k^{2}}{2 \rho_{0}}\left(\frac{\mathrm{d} \rho_{0}}{\mathrm{~d} z}\right)^{2}-\frac{1}{\rho_{0}^{3}}\left(\frac{\mathrm{d} \rho_{0}}{\mathrm{~d} z}\right)^{4}\right) u_{z 1}\right]
\end{aligned}
$$

